\title{
Mechanical thrombectomy for middle cerebral artery occlusion associated with ovarian hyperstimulation syndrome: case report and review of the literature
}

\author{
Xinxing Li ${ }^{1}$, Jihui Zheng ${ }^{2}$ \\ ${ }^{1}$ Department of Neurosurgery, Shengjing Hospital of China Medical University, Shenyang, China; ${ }^{2}$ Department of Radiology, The Fourth Affiliated \\ Hospital of China Medical University, Shenyang, China \\ Correspondence to: Jihui Zheng. Department of Radiology, The Fourth Affiliated Hospital of China Medical University, Shenyang 110004 , China. \\ Email: lixx996303@163.com.
}

\begin{abstract}
Ovarian hyperstimulation syndrome (OHSS) is a rare but serious complication of assisted reproductive technology application. The incidence of stroke symptoms associated with OHSS is lower than that of abdominal symptoms, which may occur in severe OHSS situation. If tissue plasminogen activator (tPA) is not applicable, mechanical thrombectomy (MT) is recommended for acute cerebral artery occlusion within 6 hours of symptom onset according to the current guidelines. However, the current experience and evidence is insufficient, whether acute cerebral artery occlusion associated with OHSS can be treated with MT. A 21-year-old woman underwent an ovulation induction with gonadotropin-releasing hormone agonist. Two days later, she presented with abdominal distension and diarrhea. Ascites and enlarged ovaries were confirmed by ultrasound. She was highly suspected of OHSS in by the Department of Obstetrics and Gynecology. Three days later, she presented with right-side hemiplegia, superficial coma, and aphasia, and right Babinsky sign was positive. The patient was diagnosed with left middle cerebral artery (LMCA) occlusion by computed tomography angiography (CTA). We performed emergent MT and achieved revascularization within 6 hours of cerebral ischemic symptom onset. After operation, the patient underwent various examinations, and ultrasound revealed deep vein thrombosis and right-to-left shunt due to cardiac atrial septal defect in this case. We thus reasoned that LMCA occlusion could be highly associated with OHSS. At 3-month follow-up, the patient recovered to a normal situation and was left with no neurological dysfunction. We here report MT treatment for a rare cerebral artery occlusion case associated with OHSS in right-to-left shunt patient. It is necessary to pay attention to ischemic symptoms in a severe OHSS situation in aggressive infertility treatment, especially in right-to-left shunt patients. More future studies are recommended for determining the effectiveness of the MT treatment in cerebral artery occlusion associated with OHSS.
\end{abstract}

Keywords: Artery occlusion; ovarian hyperstimulation syndrome (OHSS); mechanical thrombectomy (MT)

Submitted Jan 09, 2021. Accepted for publication Mar 03, 2021.

doi: 10.21037/apm-21-308

View this article at: http://dx.doi.org/10.21037/apm-21-308

\section{Introduction}

Ovarian hyperstimulation syndrome (OHSS) is a rare complication associated with assisted reproductive technology. It's caused by the use of human chorionic gonadotrophin (hCG). Without HCG administration, OHSS is extremely uncommon (1). Primary risk factors may cause over response of ovarian stimulation, such as young age, a history of elevated response to gonadotrophins, previous OHSS, and polycystic ovary syndrome (PCOS) (2). The prevalence of OHSS ranges between $1 \%$ and $5 \%$ and ranges in severity from moderate to severe (3-7). The incidence of cerebral artery occlusion 
in OHSS is rare $(8,9)$. Golan reports that the conditions that constitute an early pregnancy are serious and lasting for a long time (10). The clinical manifestation of OHSS includes ovarian enlargement, ascites, hemoconcentration, and hypercoagulability, and more serious manifestations include massive hydrothorax, acute renal insufficiency, and venous thrombosis (11). Artery infarction is a catastrophic complication of OHSS, which may be related to hypercoagulable state and hemoconcentration $(12,13)$. Mechanical thrombectomy is the use of stents and catheters to quickly remove the thrombus from the large intracranial blood vessels and restores blood flow (14). Some largescale clinical trials demonstrated the safety and efficacy of mechanical thrombectomy (MT) treatment in acute ischemic stroke caused by cerebral artery occlusion but not in OHSS patients $(15,16)$. In recent reports, there are also several case reports of successful MT treatment for cerebral artery occlusion in pregnant patients. The prognosis of the pregnant woman who had MT treatment had recovered to a normal condition and was left with no neurological dysfunction at the stage after half a year of thrombectomy. Among reports, few cases have been reported that cerebral infarction is caused by OHSS in pregnant women. This is the first report of a right-to-left shunt patient who had MT treatment for cerebral artery occlusion associated with OHSS. The case report by Kuwano includes no clear information about the embolic source. To explore the possible embolic source of this case, a series of tests were performed. We performed various physiological examinations including ultrasonography of the lower limbs and echocardiography. Ultrasound suggested deep vein thrombosis of the lower limb and right-to-left shunt resulting from a cardiac atrial septal defect. The blood was in a hypercoagulable state due to the OHSS. If young women express neurological deficits, especially undergoing infertility treatment, the involvement of OHSS should be suspected. Moreover, MT treatment should be performed immediately. We report a successful MT treatment for a rare cerebral artery occlusion case associated with OHSS in a right-to-left shunt patient.

We present the following article in accordance with the CARE reporting checklist (available at http://dx.doi. org/10.21037/apm-21-308).

\section{Case presentation}

All procedures performed in studies involving human participants were in accordance with the ethical standards of the institutional and/or national research committee(s) and with the Helsinki Declaration (as revised in 2013). Written informed consent was obtained from the patient for publication of this study and any accompanying images. A 21-year-old young woman underwent the ovulation induction with gonadotropin-releasing hormone agonist. Two days after egg retrieval, she presented with abdominal distension and diarrhea. Ascites and enlarged ovaries were confirmed by ultrasound. Estradiol was $5,000 \mathrm{pg} / \mathrm{mL}$ by blood test. She was highly suspected of OHSS by the Department of Obstetrics and Gynecology. Three days later, she presented with right-side hemiplegia, superficial coma, and aphasia, and right Babinsky sign was positive. The middle cerebral artery occlusion of the patient is caused by the embolism, the onset is rapid, so the patient has no transient ischemic attack. The result of National Institutes of Health Stroke Scale (NIHSS) score was 20 points. The emergent computed tomography showed no hemorrhage in the brain (Figure 1A). Computed tomography angiography (CTA) showed acute obstruction of the left middle cerebral artery (LMCA) proximal vessel (Figure $1 B$ ). The white blood cell count was $20.2 \times 10^{9} / \mathrm{L}$ ( $92.1 \%$ neutrophils), packed cell volume was $45 \%$, prothrombin time was $13.5 \mathrm{~s}$, activation time of partial thromboplastin time was $33 \mathrm{~s}$, D-dimer was $981 \mu \mathrm{g} / \mathrm{L}$, total protein was $36.8 \mathrm{~g} / \mathrm{L}$, albumin was $20.9 \mathrm{~g} / \mathrm{L}$, sodium ion was $129 \mathrm{mmol} / \mathrm{L}$, estradiol was $5,400 \mathrm{pg} / \mathrm{mL}$, and other biochemical indicators were basically normal.

The proximal segment of the LMCA was shown to be occluded on digital subtraction angiography (DSA) (Figure 1C). Angiography showed that the distal vessel was unobstructed. A $6 \times 30 \mathrm{~mm}$ Solitaire stent was used to remove the embolus. After thrombectomy, angiography showed that the M1 segment and 3 M2 branches of middle cerebral artery were unobstructed (Figure 1D). The thrombus was mainly fibrinous according to pathology tests.

To explore the possible embolic source of this case, a series of tests were performed. There were findings related to autoimmune disease, tumor disease, coagulation disfunction, collagen diseases, or atrial fibrillation. The blood was in a hypercoagulable state due to the OHSS. Ultrasound suggested deep vein thrombosis of the lower limb (Figure $2 A$ ) and right-to-left shunt resulting from a cardiac atrial septal defect (Figure 2B).

The patient's clinical symptoms improved quickly 1 day after MT. The patient was in a clear state of mind and could speak. Right-side manual muscle testing score was improved to level III. The patient was discharged with mild aphasia and 

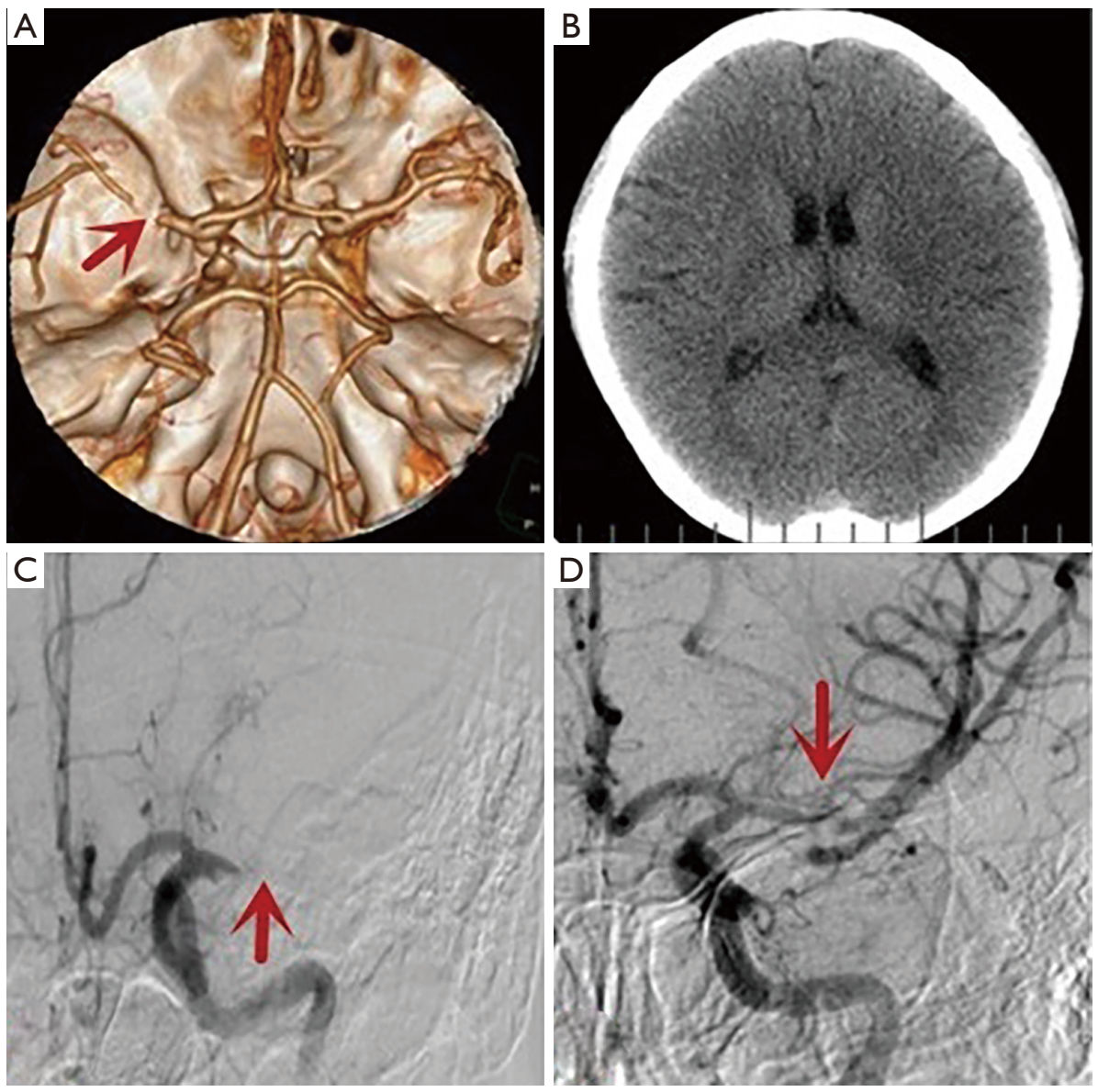

Figure 1 CT imaging of the patient in ER and DSA imaging of MT. (A) CT scan showing the proximal LMCA cutoff (arrow). (B) The CT scan showing no hemorrhage in the brain. (C) DSA imaging of the patient prior to MT proximal LMCA occlusion (arrow). (D) DSA imaging post MT showing revascularization of the M1 segment and 3 branches of M1 (arrow). CT, computed tomography; ER, emergency room; DSA, digital subtraction angiography; MT, mechanical thrombectomy; LMCA, left middle cerebral artery.
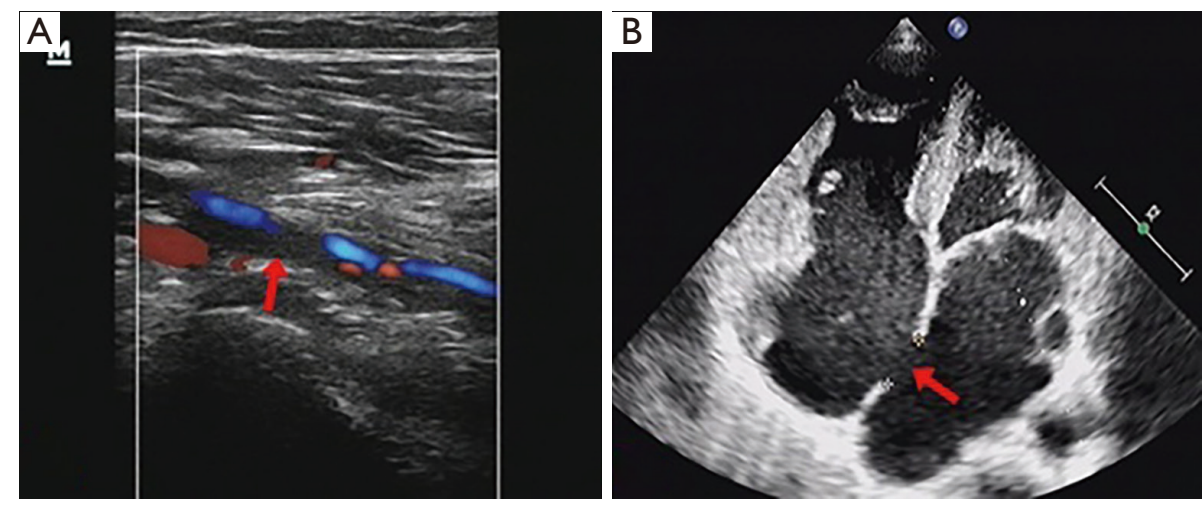

Figure 2 Ultrasound (A) and echocardiography (B) of the patient's lower limb. (A) Ultrasound showing hypoechoic thrombus in the popliteal vein (arrow). (B) Echocardiography showing cardiac atrial septal defect (arrow). 

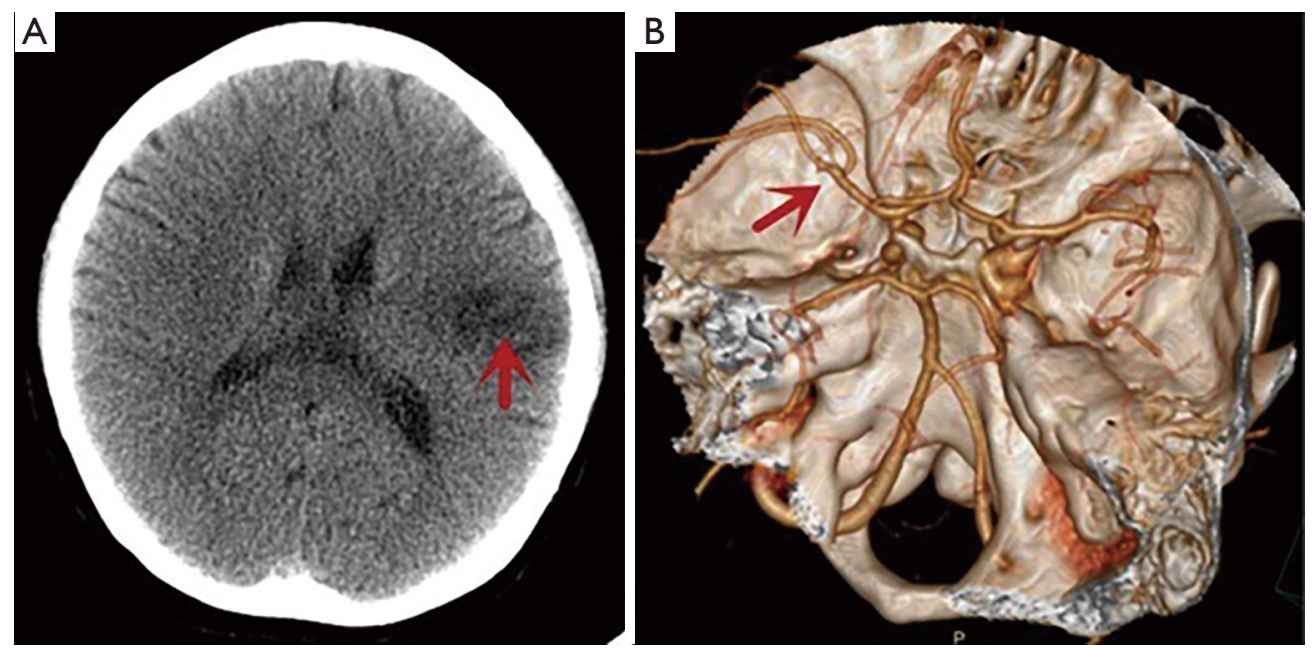

Figure 3 Follow-up CT scan of the patient. (A) A 3-month follow-up CT scan showing a lesion of small infarction in the LMCA area (arrow). (B) A 3-month follow-up CTA scan showing that the LMCA is not narrow or obstructed (arrow). CT, computed tomography; CTA, computed tomography angiography; LMCA, left middle cerebral artery.

a modified Rankin Scale (mRS) score of 1. In next follow-up appointment, 3 months after MT, the patient had recovered to a normal condition and was left with no neurological dysfunction. Follow-up CT scans showed a lesion of small infarction (Figure $3 A$ ), and CTA scans showed that the LMCA was not obstructed or stenosed (Figure 3B).

\section{Discussion and conclusions}

The pathophysiological characteristic of OHSS a can include ovarian enlargement and increased vascular permeability, which could cause pleural effusion, ascites, hemoconcentration, hypercoagulability, and electron imbalance $(11,12,17)$. Data from previous studies indicate that young age, lower BMI, and infertility are risk factors for OHSS (18-20). Vascular endothelial growth factor (VEGF), a potent stimulator of the vascular endothelium, plays an integral role in follicular growth and ovarian angiogenesis, the concentration of which has been to be related to the development and severity of the syndrome $(21,22)$. Cerebral artery infarction is more associated with serious OHSS. Patients with severe OHSS should be managed with systemic therapy, including analgesia, fluid balance, thromboprophylaxis, and management of ascites and effusion $(23,24)$. Aspirin alleviates the activation of platelets due to VEGF and blood hypercoagulability (25), a treatment which is also recommended in the therapy of severe OHSS patients and proximal large artery occlusion (26).
The patient discussed here underwent a range of examinations. Deep vein thrombosis was found in the in lower limbs, and right-to-left shunt was found to be a result of a cardiac atrial septal defect. Generally, arterial thrombi are composed mainly of platelets while venous thrombi are composed of fibrin. In this case, we thought the thrombus was mainly associated with OHSS, and it was predominantly fibrinous. Thus, thrombi may from in the deep veins in the lower limbs due to the pathophysiology of OHSS and may cause cerebral artery infarction through right-to-left shunt.

According to the most recent American Heart and American Stroke Association guidelines, MT is recommended as major treatment for proximal cerebral artery occlusion (15). However, in our case, radiation exposure problems could not be ignored. The patients could have been in the early stages of pregnancy or planning to conceive. We thus needed to consult with the family and patient before the MT treatment to confirm that pregnancy was not concern.

A case reported by Kuwano et al. (8) in 2019 is the only one that shares common features with our case. Our case and Kuwano's case both underwent infertility treatment and presented OHSS symptoms, including abdominal distension and high estradiol level. The artery occlusion sites were both in the LMCA. Generally, the prognostic mRS score of patients after MT treatment can reach to 1-2 in longterm follow-up. In recent reports, there are also several case reports of successful MT treatment for cerebral artery occlusion in pregnant patients $(27,28)$. The prognosis of the 
pregnant woman who had thrombectomy had recovered to a normal condition and was left with no neurological dysfunction at the stage after half a year of thrombectomy. Among reports, few reports have been found that OHSS is considered to be the cause of cerebral infarction in pregnant women. This is the first report of a case of successful MT for cerebral artery occlusion associated with OHSS in a right-to-left shunt patient. The case report by Kuwano includes no clear information about the embolic source. To explore the possible embolic source of this case, a series of tests were performed. We performed various physiological examinations including ultrasonography of the lower limbs and echocardiography. Ultrasound suggested deep vein thrombosis of the lower limb and right-to-left shunt resulting from a cardiac atrial septal defect. The blood was in a hypercoagulable state due to the OHSS. It is necessary to suspect OHSS involvement if young women, especially those on infertility treatment, show neurological deficits. In addition, even if the patient is a pregnant woman, if it is indicated, we should perform thrombectomy immediately.

To our knowledge, this is the first report of a case of successful MT for cerebral artery occlusion associated with OHSS in a right-to-left shunt patient. This case tells us that this phenomenon may become more common due to the development and proliferation of assisted reproductive technologies. We need to pay more attention to neurological deficit symptoms in young women, especially in infertility treatment. In addition, even if the patient is a pregnant woman, once the patient is found to have occlusion of the large blood vessels in the brain, a thrombectomy should be performed immediately. More cases need to be observed to explore the effectiveness of the MT treatment in cerebral artery occlusion associated with OHSS.

\section{Acknowledgments}

Funding: This work is supported by grants from the Liaoning Science and Technology Plan Project (no. 2018225094) and the Natural Science Foundation of Liaoning Province (no. 2020-MS-165).

\section{Footnote}

Reporting Checklist: The authors have completed the CARE reporting checklist. Available at http://dx.doi.org/10.21037/ apm-21-308

Conflicts of Interest: Both authors have completed the
ICMJE uniform disclosure form (available at http://dx.doi. org/10.21037/apm-21-308). The authors have no conflicts of interest to declare.

Ethical Statement: Both authors are accountable for all aspects of the work in ensuring that questions related to the accuracy or integrity of any part of the work are appropriately investigated and resolved. All procedures performed in studies involving human participants were in accordance with the ethical standards of the institutional and/or national research committee(s) and with the Helsinki Declaration (as revised in 2013). Written informed consent was obtained from the patient for publication of this study and any accompanying images.

Open Access Statement: This is an Open Access article distributed in accordance with the Creative Commons Attribution-NonCommercial-NoDerivs 4.0 International License (CC BY-NC-ND 4.0), which permits the noncommercial replication and distribution of the article with the strict proviso that no changes or edits are made and the original work is properly cited (including links to both the formal publication through the relevant DOI and the license). See: https://creativecommons.org/licenses/by-nc-nd/4.0/.

\section{References}

1. Kumar P, Sait SF, Sharma A, et al. Ovarian hyperstimulation syndrome. J Hum Reprod Sci 2011;4:70-5.

2. Lee TH, Liu CH, Huang CC, et al. Serum anti-Müllerian hormone and estradiol levels as predictors of ovarian hyperstimulation syndrome in assisted reproduction technology cycles. Hum Reprod 2008;23:160-7.

3. Derex L, Cho TH. Mechanical thrombectomy in acute ischemic stroke. Rev Neurol (Paris) 2017;173:106-13.

4. Steward RG, Lan L, Shah AA, et al. Oocyte number as a predictor for ovarian hyperstimulation syndrome and live birth: an analysis of 256,381 in vitro fertilization cycles. Fertil Steril 2014;101:967-73.

5. Jayaprakasan K, Chan Y, Islam R, et al. Prediction of in vitro fertilization outcome at different antral follicle count thresholds in a prospective cohort of 1,012 women. Fertil Steril 2012;98:657-63.

6. Kahnberg A, Enskog A, Brannstrom M, et al. Prediction of ovarian hyperstimulation syndrome in women undergoing in vitro fertilization. Acta Obstet Gynecol Scand 2009;88:1373-81. 
7. Grossman LC, Michalakis KG, Browne H, et al. The pathophysiology of ovarian hyperstimulation syndrome: an unrecognized compartment syndrome. Fertil Steril 2010;94:1392-8.

8. Kuwano A, Kubota Y, Nonaka T, et al. Mechanical Thrombectomy for Middle Cerebral Artery Occlusion Suspected of Ovarian Hyperstimulation Syndrome: Case Report and Review of the Literature. World Neurosurg 2019;132:300-2.

9. Abramov Y, Elchalal U, Schenker JG. Severe OHSS: An 'epidemic' of severe OHSS: a price we have to pay? Hum Reprod 1999;14:2181-3.

10. Golan A, Ronel R, Herman A, et al. Ovarian hyperstimulation syndrome: an update review. Obstet Gynecol Surv 1989;44:430-40.

11. Szabó G, Árokszállási A, Pócsi D, et al. Pathophysiology and current clinical approach of ovarian hyperstimulation syndrome. Orv Hetil 2018;159:1390-8.

12. Namavar Jahromi B, Parsanezhad ME, Shomali Z, et al. Ovarian Hyperstimulation Syndrome: A Narrative Review of Its Pathophysiology, Risk Factors, Prevention, Classification, and Management. Iran J Med Sci 2018;43:248-60.

13. Kasum M, Danolic D, Oreskovic S, et al. Thrombosis following ovarian hyperstimulation syndrome. Gynecol Endocrinol 2014;30:764-8.

14. Berkhemer OA, Fransen P, Meumer D, et al. A randomized trial of intraarterial treatment for acute ischemic stroke. $\mathrm{N}$ Engl J Med 2015;372:11-20.

15. Powers WJ, Rabinstein AA, Ackerson T, et al. 2018 Guidelines for the Early Management of Patients With Acute Ischemic Stroke: A Guideline for Healthcare Professionals From the American Heart Association/ American Stroke Association. Stroke 2018;49:e46-110.

16. Jadhav AP, Desai SM, Kenmuir CL, et al. Eligibility for Endovascular Trial Enrollment in the 6- to 24-Hour Time Window: Analysis of a Single Comprehensive Stroke Center. Stroke 2018;49:1015-7.

17. Calabro RS, Gervasi G, Leo A, et al. Neurovascular complications of ovarian hyperstimulation syndrome (OHSS): from pathophysiology to recent treatment

Cite this article as: Li X, Zheng J. Mechanical thrombectomy for middle cerebral artery occlusion associated with ovarian hyperstimulation syndrome: case report and review of the literature. Ann Palliat Med 2021;10(3):3504-3509. doi: 10.21037/ apm-21-308 options. Recent Pat Endocr Metab Immune Drug Discov 2014;8:109-16.

18. Luke B, Brown MB, Morbeck DE, et al. Factors associated with ovarian hyperstimulation syndrome (OHSS) and its effect on assisted reproductive technology (ART) treatment and outcome. Fertil Steril 2010;94:1399-404.

19. Sousa M, Cunha M, Silva JT, et al. Ovarian hyperstimulation syndrome: a clinical report on 4894 consecutive ART treatment cycles. Reprod Biol Endocrinol 2015;13:66.

20. Aramwit P, Pruksananonda K, Kasettratat N, et al. Risk factors for ovarian hyperstimulation syndrome in Thai patients using gonadotropins for in vitro fertilization. Am J Health Syst Pharm 2008;65:1148-53.

21. Levin ER, Rosen GF, Cassidenti DL, et al. Role of vascular endothelial cell growth factor in Ovarian Hyperstimulation Syndrome. J Clin Invest 1998;102:1978-85.

22. Geva E, Jaffe RB, Role of vascular endothelial growth factor in ovarian physiology and pathology. Fertil Steril 2000;74:429-38.

23. Alper MM, Smith LP, Sills ES. Ovarian hyperstimulation syndrome: current views on pathophysiology, risk factors, prevention, and management. J Exp Clin Assist Reprod 2009;6:3.

24. Nelson SM. Prevention and management of ovarian hyperstimulation syndrome. Thromb Res. 2017 Mar;151 Suppl 1:S61-4.

25. Várnagy A, Bódis J, Mánfai Z, et al. Low-dose aspirin therapy to prevent ovarian hyperstimulation syndrome. Fertil Steril 2010;93:2281-4.

26. Moulin S, Leys D. Management of acute cerebral ischaemia. Presse Med 2016;45:e451-5.

27. Sachar P, Rajamani K. Young Ischemic Stroke in Association with Ovarian Hyperstimulation Syndrome. J Stroke Cerebrovasc Dis 2016;25:e134-40.

28. Bartkova A, Sanak D, Dostal J, et al. Acute ischaemic stroke in pregnancy: a severe complication of ovarian hyperstimulation syndrome. Neurol Sci 2008;29:463-6.

(English Language Editor: J. Gray) 